\title{
A PARALLEL ALGORITHM FOR FINDING CONGRUENT REGIONS*
}

\author{
ChIn-LAung Lei and Horng-Twu Liaw \\ Department of Electrical Engineering, National Taiwan University, Taipei, Taiwan 106, R.O.C.
}

\begin{abstract}
In this paper, we study the problem for finding all the regions, which are congruent to a testing region $R$, in an input planar figure $F$. In a shared memory system with $m$ processors, we propose an efficient $\operatorname{MAX}\{O(m n), O(n \log n)\}$ time parallel algorithm, where $n, m$ are the numbers of edges of $F$ and $R$, respectively. Furthermore, our algorithm does not require to read from or write into the same memory location simultaneously, hence it can be implemented on an exclusive-read, exclusive-write (EREW) model.
\end{abstract}

\section{INTRODUCTION}

Finding the congruent regions among geometric objects is a popular topic in computational geometry. In general, this problem arises in pattern recognition, computer vision, etc. Recently, some researchers have devoted themselves to investigating this problem [1-4].

Roughly speaking, two planar regions $R$ and $S$ are congruent if there exists a mapping, including a proper geometrical translation and/or rotation, which makes $R$ onto $S$. A formal definition will be given in Section 2. In [3], they defined the congruent regions finding problem as follows: Given a planar figure $F$ and a testing region $R$, determine whether $R$ is congruent to any region of $F$, and then find all of them if they indeed exist. Figure 1 shows an example of this finding problem. Only the shadow regions bounded by edges $\left(v_{0}\right.$, $\left.v_{7}\right),\left(v_{7}, v_{8}\right),\left(v_{8}, v_{9}\right),\left(v_{9}, v_{0}\right)$ and bounded by $\left(v_{1}\right.$, $\left.v_{2}\right),\left(v_{2}, v_{3}\right),\left(v_{3}, v_{4}\right),\left(v_{4}, v_{1}\right)$ are congruent to the testing region $R$. The value labeled with each edge represents the length of that edge.

The investigation of VLSI technology has made progress in parallel operation that reveals a high degree of parallelism in multiprocessor systems. Basically, there are two different architectural models for multiprocessor systems. One of them is a tightly-coupled system where communication is through a shared memory. Thus, we also say that this system is a sharedmemory multiprocessor system. The other one is a loosely-coupled system where communication is done via an interconnection network, that is, this is a message-passing multiprocessor system.

In a shared-memory parallel system, each processor can read from or write into any memory location, depending on whether concurrent read from or concurrent write into a memory is allowed or not. Therefore, a shared-memory parallel system can be further divided into the following four models:

1. Exclusive-Read, Exclusive-Write (EREW) model. No two processors are allowed to read from or write into the same memory location simultaneously.

2. Concurrent-Read, Exclusive-Write (CREW) model.

* This work was supported in part by the National Science Council of the Republic of China under Grant NSC 81-0416E-002-20.
Processors are allowed to read from the same memory location, but no two processors are allowed to write into the same memory location simultaneously.

3. Exclusive-Read, Concurrent-Write (ERCW) model. Processors are allowed to write into the same memory location but no two processors are allowed to read from the same memory location simultaneously.

4. Concurrent-Read, Concurrent-Write (CRCW) model. Multiple processors are allowed to read from and write into the same memory location simultaneously.

Among the schemes[1-4] mentioned above, only the method proposed in [3] adopts a parallel approach to solve this problem. In this paper, we propose a method rather in EREW model than in CREW model to find the congruent regions. Our algorithm requires only $\operatorname{MAX}\{O(m n), O(n \log n)\}$ computation time, where $n, m$ are the numbers of edges of the input planar figure $F$ and the testing region $R$, respectively. Furthermore, Shih, Lee, and Yang's [3] results may contain some repetitive congruent regions. In our algorithm, we have solved this problem. The rest of this paper is organized as follows: In Section 2, some essential definitions and notations of the geometric objects are described. In Section 3, we propose an efficient parallel algorithm for finding the congruent regions and analyze the time complexity. Finally, concluding remarks are given in Section 4.

\section{PRELIMINARIES}

Before we embark on our study of an efficient parallel algorithm for finding congruent regions based on a shared-memory system, we first give some definitions, notations, and properties of the geometric objects.

A graph $G=(V, E)$ consists of a set $V$ of elements called vertices and a set $E$ of unordered pairs of members of $V$ called edges. The vertices of the graph are shown as points, while the edges are shown as lines connecting pairs of points. The edge between the pair of vertices $v_{a}$ and $v_{b}$ is denoted by $\left(v_{a}, v_{b}\right)$. Here, we call the vertices $v_{a}$ and $v_{b}$ the endpoints of the edge $\left(v_{a}, v_{b}\right)$, and we say the edge $\left(v_{a}, v_{b}\right)$ is incident with the vertices $v_{a}$ and $v_{b}$. In addition, if an edge does not 


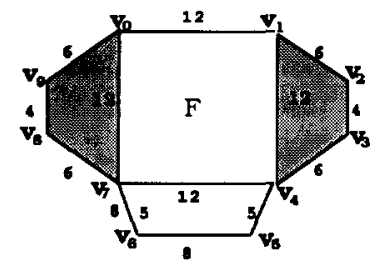

Fig. 1. The input figure $F$ and the testing region $R$.

exhibit a direction, it is called an undirected edge. Thus, an undirected edge $e=\left(v_{a}, v_{b}\right)$ can also be represented by $\left(v_{b}, v_{a}\right)$, that is, $\left(v_{a}, v_{b}\right)=\left(v_{b}, v_{a}\right)$. On the other hand, a directed edge has only one direction. Let $\vec{e}$ $=\left\langle v_{a}, v_{b}\right\rangle$ be a directed edge, where $v_{a}$ is the tail vertex and $v_{b}$ is the head vertex. Therefore, $\left\langle v_{a}, v_{b}\right\rangle \neq\left\langle v_{b}\right.$, $\left.v_{a}\right\rangle$. A path of directed edges is a sequence of directed edges that are connected with their endpoints. Without loss of generality, let $P=\left[\vec{e}_{1}, \vec{e}_{2}, \ldots, \vec{e}_{n}\right]$ denote a path of directed edges, where $\vec{e}_{1}=\left\langle v_{1}, v_{2}\right\rangle, \vec{e}_{2}=\left\langle v_{2}\right.$, $\left.v_{3}\right\rangle, \ldots, \vec{e}_{n}=\left\langle v_{n}, v_{n+1}\right\rangle$. Furthermore, a directed cycle is a path of directed edges $P=\left[\vec{e}_{1}, \vec{e}_{2}, \ldots\right.$, $\left.\vec{e}_{m}\right]$, where $\vec{e}_{1}=\left\langle v_{1}, v_{2}\right\rangle, \vec{e}_{2}=\left\langle v_{2}, v_{3}\right\rangle, \ldots, \vec{e}_{m}$ $=\left\langle v_{m}, v_{1}\right\rangle$, and $v_{i} \neq v_{j}$ when $i \neq j$. Besides, a region consists of a directed cycle and the interior of directed cycle, which is the right-hand side when we walk along the directed edge clockwisely. Here, we renewedly define the angle of each directed edge. For simplicity, let $\vec{e}=\left\langle v_{1}, v_{2}\right\rangle$. First, we translate this directed edge and let the tail vertex $v_{1}$ be at the Cartesian coordinate origin, that is, the tail vertex $v_{1}$ is regarded as a Cartesian coordinate origin. The angle between $x$-axis and $\vec{e}$ (or simply the angle of $\vec{e}$ ) is defined as the angle lying to the right of us when we walk along the positive $x$-axis to the tail of $\vec{e}$. In this paper, we use $A(\vec{e})$ to denote it. Figure 2 shows the edge $\vec{e}=\left\langle v_{1}, v_{2}\right\rangle$ and the angle $A(\vec{e})$. In addition, the angle between two adjacent edges $\vec{e}_{1}=\left\langle v_{1}, v_{2}\right\rangle$ and $\vec{e}_{2}=\left\langle v_{2}, v_{3}\right\rangle$, denoted by $A\left(\vec{e}_{1}, \vec{e}_{2}\right)$, is defined as the angle lying to the right of us when we walk along $\vec{e}_{1}$ to the tail of $\vec{e}_{2}$. Furthermore, let $\vec{e}_{1}^{\prime}=\left\langle v_{2}, v_{1}\right\rangle$ be the opposite direction of $\vec{e}_{1}$. Thus, $A\left(\vec{e}_{1}\right)$ and $A\left(\vec{e}_{1}^{\prime}\right)$ differs by $180^{\circ}$, that is, $A\left(\vec{e}_{1}^{\prime}\right)$ $=A\left(\vec{e}_{1}\right)-180$ or $A\left(\vec{e}_{1}^{\prime}\right)=A\left(\vec{e}_{1}\right)+180^{\circ}$. Without loss of generality, we assume that any angle is nonnegative and less than $360^{\circ}$. Thus, $A\left(\vec{e}_{1}^{\prime}\right)=\left(A\left(\vec{e}_{1}\right)\right.$ $-180) \bmod 360^{\circ} . A\left(\vec{e}_{1}, \vec{e}_{2}\right)$, the angle between $\vec{e}_{1}$ and $\vec{e}_{2}$, can be obtained by the following theorem.

Theorem 1. Let $\vec{e}_{1}=\left\langle v_{1}, v_{2}\right\rangle$, and $\vec{e}_{2}=\left\langle v_{2}, v_{3}\right\rangle$, then $A\left(\vec{e}_{1}, \vec{e}_{2}\right)=\left(A\left(\vec{e}_{2}\right)-A\left(\vec{e}_{1}^{\prime}\right)\right) \bmod 360^{\circ}$.
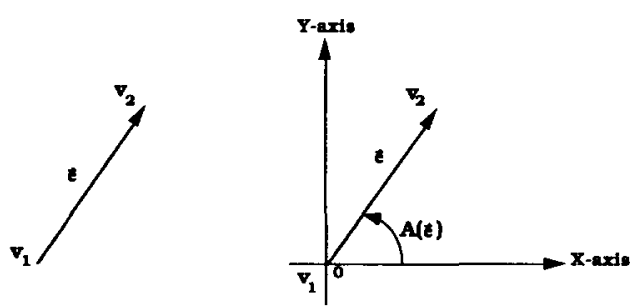

Fig. 2(a). The directed edge $\vec{e}=\left\langle v_{1}, v_{2}\right\rangle$; (b) The angle $A(\bar{e})$ of the directed edge $\bar{e}$.

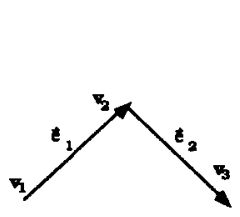

(a)

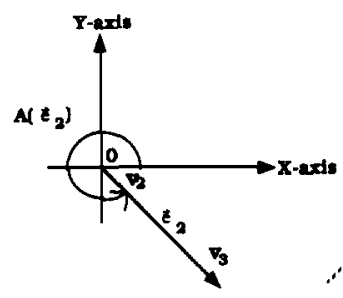

(c)

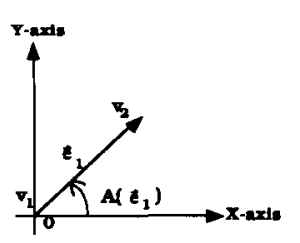

(b)

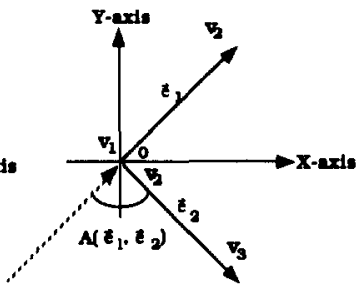

(d)
Fig. 3(a). The adjacent edges $\vec{e}_{1}$ and $\vec{e}_{2} ;$ (b) the angle $A\left(\vec{e}_{1}\right)$ of the directed edge $\vec{e}_{i} ;$ (c) The angle $A\left(\vec{e}_{2}\right)$ of the directed edge $\vec{e}_{2}$; (d) the angle $A\left(\vec{e}_{1}, \vec{e}_{2}\right)$ between the adjacent edges $\vec{e}_{1}$ and $\vec{e}_{2}$.

Proof. Since $\vec{e}_{1}$ and $\vec{e}_{2}$ intersect at the vertex $v_{2}$, the angle between them is equal to the angle between $\vec{e}_{1}^{\prime}$ and $\vec{e}_{2}$, that is, $A\left(\vec{e}_{1}, \vec{e}_{2}\right)=A\left(\vec{e}_{2}\right)-A\left(\vec{e}_{1}^{\prime}\right)$. From our assumption, any angle must be nonnegative and less than $360^{\circ}$. Hence, $A\left(\vec{e}_{1}, \vec{e}_{2}\right)=\left(A\left(\vec{e}_{2}\right)\right.$ - $\left.A\left(\vec{e}_{1}^{\prime}\right)\right) \bmod 360^{\circ}$.

Theorem 2. If $A\left(\vec{e}_{1}, \vec{e}_{2}\right)$ and $A\left(\vec{e}_{1}\right)$ are given, then $A\left(\vec{e}_{2}\right)=\left(A\left(\bar{e}^{\prime} 1\right)+A\left(\bar{e}_{1}, \bar{e}_{2}\right)\right) \bmod 360^{\circ}$.

Proof. From theorem 1, $A\left(\vec{e}_{1}, \vec{e}_{2}\right)=\left(A\left(\vec{e}_{2}\right)\right.$ - $\left.A\left(\vec{e}_{1}^{\prime}\right)\right) \bmod 360^{\circ}$. Thus, $A\left(\vec{e}_{2}\right)-A\left(\vec{e}_{1}^{\prime}\right)=A\left(\vec{e}_{1}\right.$, $\left.\vec{e}_{2}\right)+360 k$, for some integer $k$. That is, $A\left(\vec{e}_{2}\right)=$ $A\left(\vec{e}_{1}^{\prime}\right)+A\left(\vec{e}_{1}, \vec{e}_{2}\right)+360 k$. Since $A\left(\vec{e}_{2}\right)$ is also nonnegative and less than $360^{\circ}, A\left(\vec{e}_{2}\right)=\left(A\left(\vec{e}_{1}^{\prime}\right)\right.$ $+\left(A\left(\vec{e}_{1}, \vec{e}_{2}\right)\right) \bmod 360^{\circ}$.

Figure 3 shows the adjacent edges $\vec{e}_{1}=\left\langle v_{1}, v_{2}\right\rangle$, $\vec{e}_{2}=\left\langle v_{2}, v_{3}\right\rangle$ and the angles of $A\left(\vec{e}_{1}\right), A\left(\vec{e}_{2}\right), A\left(\vec{e}_{1}\right.$, $\left.\vec{e}_{2}\right)$. Furthermore, we use $|\vec{e}|$ to represent the length of any directed edge $\vec{e}$ in this paper.

Let $\vec{e}_{a}, \vec{e}_{b}, \vec{e}_{x}$, and $\vec{e}_{y}$ be four directed edges and the head vertices of $\vec{e}_{a}$ and $\vec{e}_{x}$ be the tail vertices of $\vec{e}_{b}$ and $e_{y}$, respectively. If there exists a condition such that $\left|\vec{e}_{a}\right|=\left|\vec{e}_{x}\right|,\left|\vec{e}_{b}\right|=\left|\vec{e}_{y}\right|$ and $A\left(\vec{e}_{a}, \vec{e}_{b}\right)=A\left(\vec{e}_{x}, \vec{e}_{y}\right)$, then we say that $\vec{e}_{a}$ is connected forwardly to $\vec{e}_{b}$ with respect to $\vec{e}_{x}$ and $\vec{e}_{y}$. Besides, if $\vec{e}_{a}$ is connected forwardly to $\vec{e}_{b}$, then we also say that $\vec{e}_{b}$ is connected backwardly to $\vec{e}_{a}$. Furthermore, if two regions $A$ $=\left[\vec{e}_{A_{0}}, \vec{e}_{A_{1}}, \ldots, \vec{e}_{A_{m-1}}\right]$ and $B=\left[\vec{e}_{B_{0}}, \vec{e}_{B_{1}}, \ldots\right.$, $\vec{e}_{B_{m-1}}$ ] are congruent, then there must exist some $j$ such that $\vec{e}_{A_{(i \bmod m)}}$ is connected forwardly to $\vec{e}_{A_{(i+1) \bmod m)}}$ with respect to $\vec{e}_{B_{((i+j) \bmod m)}}$ and $\vec{e}_{B_{((i+j+1) \bmod m)}}$, where $0 \leq i, j$ $\leq m-1$.

In this paper, our approach is inspired by the method found in [3] but improves upon it. In the next section, we present an efficient parallel algorithm for finding all the congruent regions. In [3], they have shown that a path of directed edges $\left[\vec{e}_{0}, \vec{e}_{1}, \ldots, \vec{e}_{m-1}\right]$ with $\left|\vec{e}_{1}\right|$ $=\left|\vec{t}_{i}\right|, 0 \leq i \leq m-1$, and $A\left(\vec{e}_{j}, \vec{e}_{j+1}\right)=A\left(\vec{t}_{j}\right.$, 
$\left.\vec{t}_{j+1}\right), 0 \leq j \leq m-2$, defines a region that is congruent to a testing region $\left[\vec{t}_{0}, \vec{t}_{1}, \ldots, \vec{t}_{m-1}\right]$. Thus, the kernel part of our proposed algorithm is to judge whether the specified candidate region from the input planar figure $F$ and the testing region $R$ possess the same edges and angles.

\section{AN EFFICIENT PARALLEL ALGORITHM}

We are now ready to propose a parallel algorithm on an EREW shared-memory computer. The algorithm presented as algorithm Parallel-Congruent makes the following assumptions. Throughout this paper, we use $m$ processing elements (PEs) to execute our algorithm, where $m$ is the number of edges of testing region $R$. Initially, we duplicate any edge of the input planar figure $F=\left\{e_{0}, e_{1}, \ldots, e_{n-1}\right\}$ into two directed edges with opposite direction. Thus, we get a new set $D=\left\{\vec{e}_{0}, \vec{e}_{1}, \ldots, \vec{e}_{2 n-1}\right\}$. In addition, the edge $\vec{t}_{i}$ of the testing region $R=\left[\vec{t}_{0}, \vec{t}_{1}, \ldots, \vec{t}_{m-1}\right]$ is stored into $P E_{i}$, where $i=0,1, \ldots, m-1$. After some steps of our algorithm, there are $m$ sets of directed edges $D_{0}$, $D_{1}, \ldots, D_{m-1}$ stored in shared memory, where $D_{j}$ $=\left\{\overrightarrow{\boldsymbol{e}}_{i}:\left|\overrightarrow{\boldsymbol{e}}_{i}\right|=\left|\vec{t}_{j}\right|, 0 \leq i \leq 2 n-1\right\}, 0 \leq j \leq m-1$. Furthermore, each element of $D_{j}$ is denoted as $D_{j}[k]$, $1 \leq k \leq n_{j}$, where $n_{j}$ is the number of edges in $D_{j}$ and each $D_{j}[k]$ contains six fields EDGE, TAIL, HEAD, ANGLE, FLINK, and BLINK. The fields TAIL and HEAD store the tail vertex and head vertex of the edge, respectively. The field ANGLE stores the angle of the edge in $D_{j}$. The field FLINK is pointed to $D_{j+1}$, BLINK is pointed to $D_{j-1}$. If $D_{j}[k]$.FLINK and $D_{j}[k]$. BLINK are pointed to an element of $D_{j+1}$ and $D_{j-1}$, respectively, then $D_{j}[k]$.EDGE can be forwardly and backwardly connected to $D_{j+1}\left[D_{j}[k]\right.$.FLINK].EDGE and $D_{j-1}\left[D_{j}[k]\right.$.BLINK].EDGE, respectively. In the following algorithm, we need to sort the order of a pair sequence. For convenience, we define the order of any two pairs as follows: $(a, b)<(c, d)$ if and only if $a$ $<c$ or $(a=c$ and $b<d)$. Without loss of generality, we assume that $m$ is even and $D_{j}[k]$.FLINK and $D_{j}[k]$.BLINK are null initially. The algorithm, described in what follows as algorithm Parallel-Congruent proceeds in stages.

\section{Algorithm Parallel-Congruent}

Input: The set of edges $E=\left\{e_{0}, \ldots, e_{n-1}\right\}$ of the input planar figure $F$ and the cycle of directed edges $\left[\bar{t}_{0}, \ldots, \vec{t}_{m-1}\right]$ of the testing region $R$.

Output: All the regions of planar figure $F$ that are congruent to the testing region $R$.

Step 1: Duplicate the set of edges $E=\left\{e_{0}, e_{1}, \ldots\right.$, $\left.e_{n-1}\right\}$ of $F$ to the set of directed edges $D=$ $\left\{\vec{e}_{0}, \vec{e}_{1}, \ldots, \vec{e}_{2 n-1}\right\}$.

Step 2: Load $\vec{t}_{j}$ into the processing element $P E_{j}$, where $j=0,1, \ldots, m-1$.

Step 3: Calculate the values $F A_{i}=A\left(\vec{t}_{i}, \vec{t}_{(i+1) \bmod m}\right)$ and store it into $P E_{i}$, where $i=0,1, \ldots$, $m-1$.
Step 4: Load the set of directed edges $D=\left\{\vec{e}_{0}, \vec{e}_{1}\right.$, $\left.\ldots, \vec{e}_{2 n-1}\right\}$ into the processing elements by the following operations.

For $i=0$ to $2 n-1$ do

$$
\begin{aligned}
& \text { For } j=0 \text { to } m-1 \text { do in parallel } \\
& \quad k_{i}=(j+i) \bmod 2 n \\
& \vec{e}_{k_{j}} \text { enters } P E_{j} \\
& \quad \text { If }\left|\vec{e}_{k_{j}}\right|=\left|\vec{t}_{j}\right| \text { then } P E_{j} \text { copies } \vec{e}_{k_{j}} \text { into } \\
& \text { the set } D_{j} \\
& \text { end }
\end{aligned}
$$$$
\text { end }
$$

Step 5: For $j=0$ to $m-1$ do in parallel

Calculate the relative angle of each edge in $D_{j}$ and let the value of the angle be end $A_{j}\left(\vec{e}_{k_{j}}\right), 0 \leq k_{j} \leq 2 n-1$.

Step 6: For $j=0$ to $m-1$ do in parallel

Sort the pairs $\left(v_{\bar{e} k_{j}}, A_{j}\left(\vec{e}_{k_{j}}\right)\right), 0 \leq k_{j}$ $\leq 2 n-1$, where $v_{\bar{e}_{k_{j}}}$ is the tail vertex of edge $\vec{e}_{k_{j}}$ in $D_{j}$. end

Step 7: For $j=0,2,4, \ldots, m-2$ do in parallel

(7.1) For $k=1$ to $n_{j}$ do $/ / n_{j}$ is the number of edges in $D_{j} / /$

$A_{j}\left(\vec{e}_{k_{j}}{ }^{\prime}\right)=\left(A_{j}\left(\vec{e}_{k_{j}}\right)-180^{\circ}\right) \bmod 380^{\circ}$ $A_{j+1}\left(\vec{e}_{p_{j+1}}\right)=\left(F A_{j}+A_{j}\left(\vec{e}_{k_{j}}{ }^{\prime}\right)\right) \bmod 360^{\circ}$

$$
\begin{gathered}
/ / 0 \leq k_{j} \\
p_{j+1} \leq \\
2 n-1 / /
\end{gathered}
$$

Let this edge $\vec{e}_{k_{j}}$ be pointed by $L$ and use binary search to find the pair ( $v$, $\left.A_{j+1}\left(\vec{e}_{p_{j+1}}\right)\right)$ in $D_{j+1}$, where $v$ is both the head vertex of edge $\vec{e}_{k_{j}}$ in $D_{j}$ and the tail vertex of certain edge in $D_{j+1}$. Let $R$ be a pointer to this found edge in $D_{j+1}$. If such $R$ exists, then $D_{j}[k]$.FLINK $\leftarrow R$ and $D_{j+1}\left[D_{j}[k]\right.$. FLINK].BLINK $\leftarrow L$.

If such $R$ does not exist, then $D_{j}[k] . \mathrm{EDGE} \leftarrow 0$. end

end

Step 8: For $j=1,3,5, \ldots, m-3$ do in parallel Perform step (7.1) of Step 7.

Step 9: All processing elements perform this step iteratively until there is no more edge in each $D_{j}, 0 \leq j \leq m-1$, which can be deleted in one iteration.

(9.1) For $\mathrm{j}=0,2,4, \ldots, m-2$ do (a) and (b) in parallel

(a) For $k=1$ to $n_{j}$ do If $D_{j+1}\left[D_{j}[k]\right.$. FLINK $]$. EDGE $=0$
then $D_{j}[k]$. EDGE $\leftarrow 0$
end

(b) For $k=1$ to $n_{j+1}$ do If $D_{j}\left[D_{j+1}[k]\right.$. BLINK].EDGE $=0$ then $D_{j+1}[2 \mathrm{k}]$.EDGE $\leftarrow 0$ end

end

(9.2) For $j=1,3,5, \ldots, m-3$ do in parallel 


\author{
$P E_{j}$ and $P E_{j+1}$ simultaneously \\ perform steps (a) and (b) of step \\ 9 , respectively.
} end

Step 10: (10.1) Flink all the remainder regions into some sets $A_{0}, \ldots, A_{k}, 0 \leq k \leq 2 n-1$, in the shared memory, from $D_{0}$ to $D_{m-1}$ via appropriate pointers.

(10.2) Rotate each remainder region $A_{i}, 0$ $\leq i \leq k$, so that the smallest labeled vertex is at the first position.

(10.3) Sort $A_{0}, \ldots, A_{k}$ with the first vertex.

(10.4) Erase the repetitive regions and output all the proper congruent regions.

Now, let us discuss this algorithm. The algorithm Parallel-Congruent uses $m$ processing elements, where $m$ is the number of edges of the testing region $R$. Step 1 requires $O(n)$ time to duplicate the set of edges of the input planar figure $F$, where $n$ is the number of edges of $F$. In Step 2 , the directed edges $\left[\vec{t}_{0}, \vec{t}_{1}, \ldots\right.$, $\left.\vec{t}_{m-1}\right]$ are loaded into the processing elements that requires $O(m)$ computation time. Similarly, Step 3 requires $O(m)$ time to calculate the relative angles of all the directed edges $\left[\vec{t}_{0}, \vec{t}_{1}, \ldots, \vec{t}_{m-1}\right]$. In Step 4 , the set of directed edges $D=\left\{\vec{e}_{0}, \vec{e}_{1}, \ldots, \vec{e}_{2 n-1}\right\}$ are loaded into the processing elements and compared with the directed edge $\vec{t}_{j}$ on $P E_{j}$, where $j=0,1, \ldots, m-1$. Since there exist $2 n$ directed edges, this step requires $O(n)$ computation time. In the worst case, there exist $O(n)$ directed edges in $D_{j}$ when Step 4 is completed. In Step 5, each processing element $P E_{j}, j=0,1, \ldots$, $m-1$, calculates the relative angles of all the edges in $D_{j}$. Since there may exist $O(n)$ edges in $D_{j}$ in the worst case, it takes $O(n)$ computation time. Step 6 sorts all the pairs in $D_{j}$, where each pair is composed by a tail vertex and an angle of certain edge. From [5] we know that any algorithm for sorting $n$ elements must require at least $\Omega(n \log n)$ operations in the worst case. In this step, we sort the tail vertices in first pass, and then sort the angles in second pass. Each of these two passes is completed in $O(n \log n)$ time. Therefore, the whole step takes $O(n \log n)$ time. In Step 7, for each edge in $D_{j}, j=0,2, \ldots, m-2$, it uses binary search scheme to find the ordered pair $\left(v A_{j+1}\left(\vec{e}_{p_{j+1}}\right)\right)$ in $D_{j+1}$, where $v$ is not only the head vertex in $D_{j}$ but also the tail vertex in $D_{j+1}$, and $A_{j+1}\left(\vec{e}_{p_{j+1}}\right)$ is the angle of certain edge in $D_{j+1}, 0 \leq p_{j+1} \leq 2 n-1$. First, we adopt binary search to find $v$ in $D_{j+1}$, and then adopt binary search
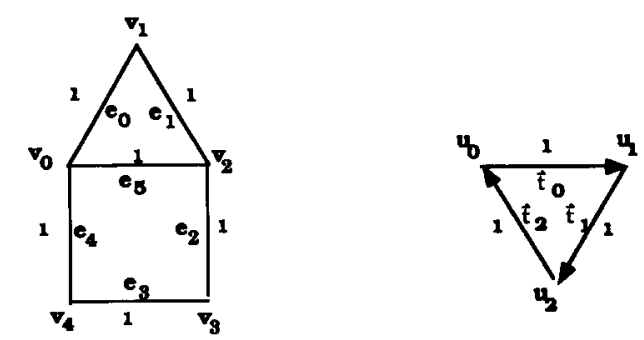

Fig. 4 (a). The input planar figure $F$; (b) The testing region $R$.
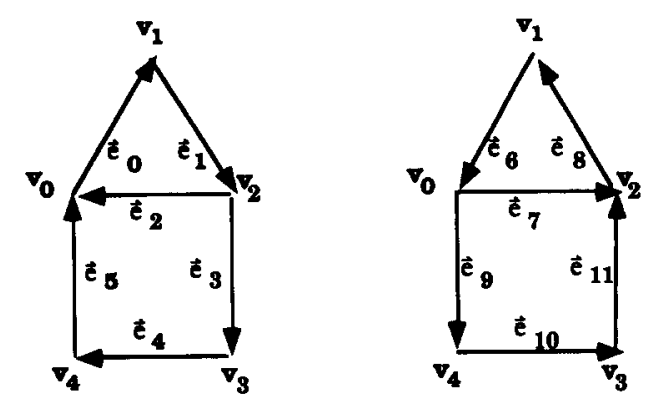

Fig. 5. All the directed edges of figure $F$.

to find $A_{j+1}\left(\vec{e}_{p_{j+1}}\right)$ in $D_{j+1}$ again. Since there may exist $O(n)$ edges in $D_{j+1}$ in the worst case, it requires $O(\log$ $n$ ) time to search the tail vertex and the angle of certain edge in $D_{j+1}$. Therefore, the whole step requires $O(n$ $\log n$ ) computation time. Step 8 is similar to Step 7, and it takes $O(n \log n)$ computation time. In Step 9, we use the concept of the odd-even transposition sort $[6,7]$ to delete some unsuitable edges in $D_{j}$. Shih, Lee, and Yang [3] have shown that this step must perform $O(\mathrm{~m})$ iterations in the worst case to complete this odd-even scheme. Therefore, this step takes $O(m n)$ computation time in the worst case. Furthermore, there may exist $O(n)$ remainder regions after Step 9. Thus, using $m$ processing elements, Step 10.1 requires $O(n)$ computation time to feed these regions into $A_{0}, \ldots$, $A_{k}, 0 \leq k \leq 2 n-1$, in shared memory. Step 10.2 requires $O(m)$ time to rotate each $A_{i}, 0 \leq i \leq k$. Thus, it takes $O(n / m)^{*} O(m)=O(n)$ computation time to complete this step. Step 10.3 sorts $A_{0}, \ldots, A_{k}$ with the first vertex. Many papers have been published on parallel sorting scheme for last three decades. Whichever we choose, this step does not exceed $O(n \log n)$ computation time. Moreover, there may still exist $O(n)$ regions after Step 10.3. Thus, it takes $O(m n)$ computation time to erase and sequentially output all the congruent regions. Consequently, $\operatorname{MAX}\{O(m n), O(n$ $\log n)\}$ time is required in Step 10 .

From the above analysis, we observe that our algorithm is dominated by Step 6, 7, 8, 9, and 10. Thus, the time complexity of this proposed algorithm is $\operatorname{MAX}\{O(m n), O(n \log n)\}$.

The following example illustrates how the congruent regions are found by the algorithm Parallel-Congruent.

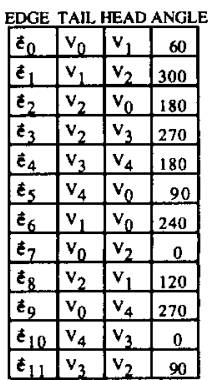

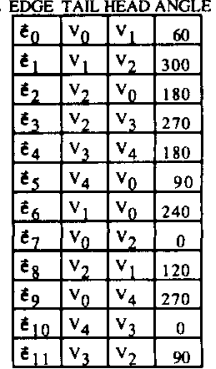

$\mathrm{D}_{2}$

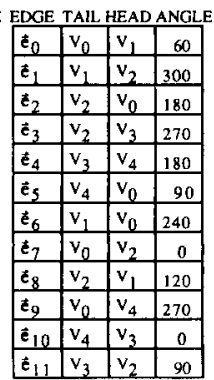

Fig. 6. The contents of $D_{0}, D_{1}$ and $D_{2}$. 
Do

$D_{1}$

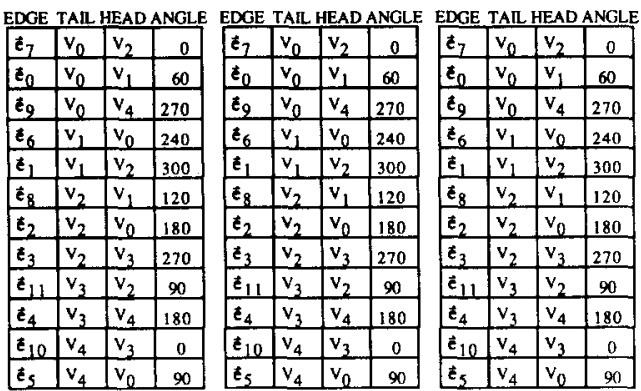

Fig. 7. The result of Fig. 6 after sorting.

\section{Example}

Figure 4(a) shows an input planar figure $F$, and Fig. 4 (b) shows a testing region $R$. By executing the Algorithm Parallel-Congruent, we easily find all the regions that are congruent to the testing region $R$. The input planar figure $F$ consists of a set of edges $E=\left\{e_{0}\right.$, $\left.e_{1}, e_{2}, e_{3}, e_{4}, e_{5}\right\}$ and the testing regions $R$ is defined as $\left[t_{0}, t_{1}, t_{2}\right]$. For simplicity, assume that $\left|e_{0}\right|=\left|e_{1}\right|$ $=\left|e_{2}\right|=\left|e_{3}\right|=\left|e_{4}\right|=\left|e_{5}\right|=\left|\vec{t}_{0}\right|=\left|\vec{t}_{1}\right|=\left|\vec{t}_{2}\right|$ $=1$.

Step 1 duplicates the set of edges $E=\left\{e_{0}, e_{1}, \ldots\right.$, $\left.e_{5}\right\}$ of the input figure $F$ to the set of directed edges $D$ $=\left\{\vec{e}_{0}, \vec{e}_{1}, \ldots, \vec{e}_{11}\right\}$. Figure 5 shows all the directed edges of figure $F$.

In Step 2, all the directed edges $\vec{t}_{0}, \vec{t}_{1}$, and $\vec{t}_{2}$ are loaded into the processing elements $P E_{0}, P E_{1}$, and $P E_{2}$, respectively.

Step 3 calculates the angle of $F A_{0}=A\left(\bar{t}_{0}, \bar{t}_{1}\right)$ $=A\left(\vec{t}_{1}\right)-\left(A\left(\vec{t}_{0}\right)-180^{\circ}+360^{\circ}\right), F A_{1}=A\left(\vec{t}_{1}\right.$, $\left.\vec{t}_{2}\right)=A\left(\vec{t}_{2}\right)-\left(A\left(\vec{t}_{1}\right)-180^{\circ}\right)$, and $F A_{2}=A\left(\vec{t}_{2}\right.$, $\left.\vec{t}_{0}\right)=A\left(\vec{t}_{0}\right)-\left(A\left(\vec{t}_{2}\right)-180^{\circ}+360^{\circ}\right)+360^{\circ}$. Without loss of generality, let $F A_{0}=240^{\circ}-\left(0-180^{\circ}\right.$ $\left.+360^{\circ}\right)=60^{\circ}, F A_{1}=120^{\circ}-\left(240^{\circ}-180^{\circ}\right)=60^{\circ}$, and $F A_{2}=0-\left(120^{\circ}-180^{\circ}+360^{\circ}\right)+360^{\circ}=60^{\circ}$. Then, we save $F A_{0}, F A_{1}$, and $F A_{2}$ into $P E_{0}, P E_{1}$, and $P E_{2}$, respectively. In Step 4 , if $\left|\vec{e}_{k_{j}}\right|=\left|\vec{t}_{j}\right|$ then $P E_{j}$ copies $\vec{e}_{k_{j}}$ into the set $D_{j}$, where $0 \leq k_{j} \leq 11,0 \leq j \leq 2$. Therefore, $D_{0}=\left\{\vec{e}_{0}, \vec{e}_{1}, \ldots, \vec{e}_{11}\right\}, D_{1}=\left\{\vec{e}_{0}, \vec{e}_{1}, \ldots\right.$
$\mathrm{D}_{0}$

BLINK EDGE TAIL HEAD ANGLE FLINK

\begin{tabular}{|c|c|l|l|c|c|}
\hline 0 & 0 & $\mathrm{~V}_{0}$ & $\mathrm{~V}_{2}$ & 0 & 0 \\
\hline 0 & $\overrightarrow{\mathrm{e}}_{0}$ & $\mathrm{~V}_{0}$ & $\mathrm{~V}_{1}$ & 60 & $\overrightarrow{\mathrm{e}}_{1}$ \\
\hline 0 & 0 & $\mathrm{~V}_{0}$ & $\mathrm{~V}_{4}$ & 270 & 0 \\
\hline 0 & 0 & $\mathrm{~V}_{1}$ & $\mathrm{~V}_{0}$ & 240 & 0 \\
\hline 0 & $\overrightarrow{\mathrm{e}}_{1}$ & $\mathrm{~V}_{1}$ & $\mathrm{~V}_{2}$ & 300 & $\overrightarrow{\mathrm{e}}_{2}$ \\
\hline 0 & 0 & $\mathrm{~V}_{2}$ & $\mathrm{~V}_{1}$ & 120 & 0 \\
\hline 0 & $\overrightarrow{\mathrm{e}}_{2}$ & $\mathrm{~V}_{2}$ & $\mathrm{~V}_{0}$ & 180 & $\overrightarrow{\mathrm{e}}_{0}$ \\
\hline 0 & 0 & $\mathrm{~V}_{2}$ & $\mathrm{~V}_{3}$ & 270 & 0 \\
\hline 0 & 0 & $\mathrm{~V}_{3}$ & $\mathrm{~V}_{2}$ & 90 & 0 \\
\hline 0 & 0 & $\mathrm{~V}_{3}$ & $\mathrm{~V}_{4}$ & 180 & 0 \\
\hline 0 & 0 & $\mathrm{~V}_{4}$ & $\mathrm{~V}_{3}$ & 0 & 0 \\
\hline 0 & 0 & $\mathrm{~V}_{4}$ & $\mathrm{~V}_{0}$ & 90 & 0 \\
\hline
\end{tabular}

$D_{1}$

BLINK EDGE TAIL HEAD ANGLE FLINK
\begin{tabular}{|c|c|c|c|c|c|}
\hline 0 & 0 & $\mathrm{~V}_{0}$ & $\mathrm{~V}_{2}$ & 0 & 0 \\
\hline $\overrightarrow{\mathrm{e}}_{2}$ & $\overrightarrow{\mathrm{e}}_{0}$ & $\mathrm{~V}_{0}$ & $\mathrm{~V}_{1}$ & 60 & $\overrightarrow{\mathrm{e}}_{1}$ \\
\hline 0 & 0 & $\mathrm{~V}_{0}$ & $\mathrm{~V}_{4}$ & 270 & 0 \\
\hline 0 & 0 & $\mathrm{~V}_{1}$ & $\mathrm{~V}_{0}$ & 240 & 0 \\
\hline $\overrightarrow{\mathrm{e}}_{0}$ & $\overrightarrow{\mathrm{e}}_{1}$ & $\mathrm{~V}_{1}$ & $\mathrm{~V}_{2}$ & 300 & $\overrightarrow{\mathrm{e}}_{2}$ \\
\hline 0 & 0 & $\mathrm{~V}_{2}$ & $\mathrm{~V}_{1}$ & 120 & 0 \\
\hline $\overrightarrow{\mathbf{e}}_{1}$ & $\overrightarrow{\mathrm{e}}_{2}$ & $\mathrm{~V}_{2}$ & $\mathrm{~V}_{0}$ & 180 & $\overrightarrow{\mathrm{e}}_{0}$ \\
\hline 0 & 0 & $\mathrm{~V}_{2}$ & $\mathrm{~V}_{3}$ & 270 & 0 \\
\hline 0 & 0 & $\mathrm{~V}_{3}$ & $\mathrm{~V}_{2}$ & 90 & 0 \\
\hline 0 & 0 & $\mathrm{~V}_{3}$ & $\mathrm{~V}_{4}$ & 180 & 0 \\
\hline 0 & 0 & $\mathrm{~V}_{4}$ & $\mathrm{~V}_{3}$ & 0 & 0 \\
\hline 0 & 0 & $\mathrm{~V}_{4}$ & $\mathrm{~V}_{0}$ & 90 & 0 \\
\hline
\end{tabular}

$\mathrm{D}_{2}$

BLINK EDGE TAIL HEAD ANGLE FLINK
\begin{tabular}{|c|c|l|l|c|c|}
\hline 0 & 0 & $\mathrm{~V}_{0}$ & $\mathrm{~V}_{2}$ & 0 & 0 \\
\hline $\overrightarrow{\mathrm{e}}_{2}$ & $\overrightarrow{\mathrm{e}}_{0}$ & $\mathrm{~V}_{0}$ & $\mathrm{~V}_{1}$ & 60 & 0 \\
\hline 0 & 0 & $\mathrm{~V}_{0}$ & $\mathrm{~V}_{4}$ & 270 & 0 \\
\hline 0 & 0 & $\mathrm{~V}_{1}$ & $\mathrm{~V}_{0}$ & 240 & 0 \\
\hline $\overrightarrow{\mathrm{e}}_{0}$ & $\overrightarrow{\mathrm{e}}_{1}$ & $\mathrm{~V}_{1}$ & $\mathrm{~V}_{2}$ & 300 & 0 \\
\hline 0 & 0 & $\mathrm{~V}_{2}$ & $\mathrm{~V}_{1}$ & 120 & 0 \\
\hline $\overrightarrow{\mathrm{e}}_{1}$ & $\overrightarrow{\mathrm{e}}_{2}$ & $\mathrm{~V}_{2}$ & $\mathrm{~V}_{0}$ & 180 & 0 \\
\hline 0 & 0 & $\mathrm{~V}_{2}$ & $\mathrm{~V}_{3}$ & 270 & 0 \\
\hline 0 & 0 & $\mathrm{~V}_{3}$ & $\mathrm{~V}_{2}$ & 90 & 0 \\
\hline 0 & 0 & $\mathrm{~V}_{3}$ & $\mathrm{~V}_{4}$ & 180 & 0 \\
\hline 0 & 0 & $\mathrm{~V}_{4}$ & $\mathrm{~V}_{3}$ & 0 & 0 \\
\hline 0 & 0 & $\mathrm{~V}_{4}$ & $\mathrm{~V}_{0}$ & 90 & 0 \\
\hline
\end{tabular}

Fig. 8. Tables of $D_{0}, D_{1}$ and $D_{2}$ that contain FLINK and BLINK 
$\left.\vec{e}_{11}\right\}$ and $D_{2}=\left\{\vec{e}_{0}, \vec{e}_{1}, \ldots, \vec{e}_{11}\right\}$. Step 5 calculates the relative angle of each edge in $D_{j}, 0 \leq j \leq 2$, and let the angle be $A_{j}\left(\vec{e}_{k_{j}}\right), 0 \leq k \leq 11$. For simplicity, we show them in terms of three tables, each table contains four fields EDGE, TAIL, HEAD, and ANGLE. The field EDGE represents the edge name in $D_{j}, 0 \leq j \leq 2$. The fields TAIL and HEAD are the tail vertex and head vertex of certain edge, respectively. Furthermore, the field ANGLE stores the relative angle. In order to continue our example, each angle in field ANGLE is given a suitable value. Figure 6 shows these three tables.

In Step 6, we sort all the pairs $\left(v_{\bar{e}_{k_{j}}}, A\left(\vec{e}_{k_{j}}\right)\right), 0 \leq k_{j}$ $\leq 11$, where $v_{\bar{e}_{k_{j}}}$ is the tail vertex of edge $\vec{e}_{k_{j}}$ in $D_{j}, 0$ $\leq j \leq 2$. Figure 7 shows the result, after sorting, of Fig. 6.

In Steps 7 and 8, binary search scheme is adopted to find the appropriate pair with respect to the testing region $R$. Two pointers FLINK and BLINK are used to point to the suitable edge, respectively. For ease and simplicity, we replace these pointers with appropriate edges. Figure 8 shows this result. Furthermore, in Step 9 , the scheme of the odd-even transposition sort is adopted to delete unsuitable edges in each $D_{j}$ at each iteration until no more edge can be deleted in each $D_{j}$.

In Step 10, all the remainder regions are loaded into three sets $A_{0}, A_{1}$, and $A_{2}$, that is, $A_{0}=\left\{\vec{e}_{0}, \vec{e}_{1}, \vec{e}_{2}\right\}$, $A_{1}=\left\{\vec{e}_{1}, \vec{e}_{2}, \vec{e}_{0}\right\}$, and $A_{2}=\left\{\vec{e}_{2}, \vec{e}_{0}, \vec{e}_{1}\right\}$. Then, these three sets $A_{0}, A_{1}$, and $A_{2}$ are rotated, respectively. Therefore, $A_{0}=\left\{\vec{e}_{0}, \vec{e}_{1}, \vec{e}_{2}\right\}, A_{1}=\left\{\vec{e}_{0}, \vec{e}_{1}, \vec{e}_{2}\right\}$, and $A_{2}=\left\{\vec{e}_{0}, \vec{e}_{1}, \vec{e}_{2}\right\}$. Now, these three sets are sorted in terms of the first vertex. Finally, we erase the possible repetitive sets and output all the remainder regions, which are congruent to the testing region $R$. Therefore, only the region bounded by edges $\vec{e}_{0}, \vec{e}_{1}$, and $\vec{e}_{2}$ is congruent to the testing region $R$.

\section{CONCLUDING REMARKS}

In this paper, we propose an efficient parallel algorithm for finding the congruent regions. Compare with previous papers. In $[8,9]$, they limit the input form to be polygons. In [3], they adopts a CREW sharedmemory model. In Step 4 of our algorithm, each processing element $P E_{j}, j=0,1, \ldots, m-1$, read the different edge $\vec{e}_{i}, i=0,1, \ldots, 2 n-1$, on the same time. Therefore, we modify their CREW model to EREW model in this paper. Furthermore, in [3], their proposed algorithm requires $O\left(n^{2}\right)$ computation time for finding the congruent regions by using $m$ processing elements. Whereas, the time complexity of our algorithm only takes $\operatorname{MAX}\{O(m n), O(n \log n)\}$ computation time using the same number of processing elements. In addition, the results in [3] may contain some repetitive congruent regions. Here, we have solved this serious problem completely in our proposed algorithm. Moreover, our algorithm can be easily modified to handle the situation where the number of available processing elements is not the same as the number of edges in the testing region. Up until now, there still existed some interesting open problems, for instance, can we propose a parallel algorithm for finding all the 3-dimensional regions, which are congruent to a planar or 3-dimensional testing regions $R$ ? In addition, there is a region similarity problem, which is similar to this congruent region problem, in computational geometry. The difference between them is that the similarity problem must also consider the scaling factors $[1,8,9,10]$.

Acknowledgement-The authors would like to thank the referees for their valuable comments and suggestions.

\section{REFERENCES}

1. H. Alt, K. Mehlhorn, H. Wagener, and E. Weizi, Congruence, similarity, and symmetrics of geometric objects In: Proc. 3rd ACM Ann. Symp. on Computational Ge. ometry, 308-315 (1987)

2. M. D. Atkinson, An optimal algorithm for geometrical congruence, Journal of Algorithms, 8, 159-172 (1987).

3. Z. C. Shih, R. C. T. Lee, and S. N. Yang, A parallel algorithm for finding congruent regions, Parallel Computing, 13, 135-142 (1990).

4. K. Sugihara, An $n \log n$ algorithm for determining the congruity of polyhedra, Journal of Computer and System Sciences 29, 36-47 (1984).

5. E. Horowitz and S. Sahni, Fundamentals of Data Structures, Computer Science Press, Rockville, MD (1976).

6. G. Baudet and D. Stevenson, Optimal sorting algorithms for parallel computers, IEEE Transactions on Computer. 27(1) 84-87 (1978).

7. D. E. Knuth, The Art of Computer Programming, Vol. 3, Sorting and Searching, Addison-Wesley, Reading, MA (1973).

8. S. G. Akl and G. T. Toussaint, An improved algorithm to check for polygon similarity, Information Processing Letters, 7(3) 127-128 (1978).

9. A. Byka, On polygon similarity, Information Processing Letters, 9(1) 23-25 (1979).

10. M. J. Atallah, Checking similarity of planar figures, International Journal of Computer Information Science, 13(4) 279-290 (1984). 